Introduction Transfusion thresholds for upper gastrointestinal bleeding (UGIB) are controversial. Observational studies suggest associations between liberal red blood cell (RBC) transfusion and adverse outcome, and a recent trial reported increased mortality following liberal transfusion.

Methods Pragmatic cluster randomised trial to evaluate the feasibility and safety of implementing a restrictive (transfusion when haemoglobin $(\mathrm{Hb})<8 \mathrm{~g} \mathrm{dL}$ ) versus liberal (transfusion when $\mathrm{Hb}<10 \mathrm{~g} / \mathrm{dL}$ ) RBC transfusion policy for UGIB. Hospitals were randomised to a policy which was implemented through a multi-faceted educational intervention targeting all staff caring for patients with UGIB. All adult patients were eligible to participate, regardless of co-morbidity; the only exclusion criterion was exsanguinating haemorrhage. Feasibility and exploratory clinical outcomes were recorded up to day 28.

Results 936 patients were enrolled in 6 hospitals. The consent rate for follow up was higher in the liberal arm (62\% vs. 55\%; $\mathrm{P}=0.04)$. There were some baseline imbalances, however the Rockall and Blatchford scores were identical between arms as was the prevalence of ischaemic heart disease (IHD) (14\% liberal arm vs. $15 \%$ restrictive arm). Protocol adherence was $96 \%$ in the restrictive arm vs. $83 \%$ in the liberal arm. In patients with a $\mathrm{Hb}<12 \mathrm{~g} / \mathrm{dL}$, the $\mathrm{Hb}$ at discharge was $10.7 \mathrm{~g} / \mathrm{dL}$ (sd 1.2) in the liberal arm vs. $10.1 \mathrm{~g} / \mathrm{dL}$ (sd 1.3) in the restrictive arm $(\mathrm{P}=$ $0.05)$. In the restrictive arm there was a $13 \%$ absolute reduction in the proportion of patients receiving RBCs $(95 \%$ CI for difference in $\%-35$ to $11, \mathrm{P}=0.23$ ) with a mean of 0.8 fewer $\mathrm{RBC}$ units transfused $(95 \% \mathrm{CI}:-1.9$-to $0.3, \mathrm{P}=0.12)$. Clinical outcomes were better in the restrictive arm, although differences were not statistically significant (28-day mortality, 7\% liberal vs. $5 \%$ restrictive, adjusted difference in $\%=-1.3,95 \%$ CI: -8.0 to $5.5, \mathrm{P}=0.63$; further bleeding, 9 vs. $5 \%, \mathrm{P}=0.29$; serious adverse events, $22 \%$ vs. $18 \%, \mathrm{P}=0.48$ ). In the subgroup with IHD, there was a large observed difference for mortality $(12 \%$ restrictive $\operatorname{arm}(n=6)$ vs. $3 \%$ liberal arm $(n=2)$; interaction $P$ $=0.11$ ).

Conclusion The protocol was feasible and generated clinically important differences in the level of anaemia and RBC exposure. There was a consistent trend towards fewer complications in the restrictive arm, apart from the increased mortality observed in patients with IHD, which could in part be explained by imbalances in baseline risk. A large trial is required to clarify the riskbenefit balance before advocating restrictive RBC transfusion for all patients with UGIB.

Disclosure of Interest None Declared.

\section{PTU-185 UPDATE ON THE HALT-IT TRIAL PROGRESS: TRANEXAMIC ACID FOR THE TREATMENT OF GASTROINTESTINAL HAEMORRHAGE - AN INTERNATIONAL, RANDOMISED, DOUBLE BLIND PLACEBO CONTROLLED TRIAL}

${ }^{1} \mathrm{~V}$ Jairath*, ${ }^{2} \mathrm{H}$ Shakur, ${ }^{2} \mathrm{P}$ Edwards, ${ }^{2} \mathrm{~K}$ Ker, ${ }^{3} \mathrm{D}$ Manno, ${ }^{4}$ I Gilmore, ${ }^{5} \mathrm{~A}$ Veitch, ${ }^{6} \mathrm{~S}$ Stanworth, ${ }^{7} \mathrm{~T}$ Coats, ${ }^{2}$ Roberts. ${ }^{1}$ Translational Gastroenterology Unit, Nuffield Department of Medicine, University of Oxford, Oxford, UK; ${ }^{2}$ Clinical Trials Unit, London School of Hygiene and Tropical Medicine, London, UK; ${ }^{3} \mathrm{Clinical}$ Trials, London School of Hygiene and Tropical Medicine, London, UK; ${ }^{4}$ Hepatology, University of Liverpool, Liverpool, UK; ${ }^{5}$ Gastroenterology, New Cross Hospital, Wolverhampton, UK; ${ }^{6}$ Transfusion, NHS Blood and Transplant, Oxford, UK; ${ }^{7}$ Emergency Medicine, University of Leicester, Leicester, UK

\subsection{6/gutjnl-2014-307263.259}

Introduction Gastrointestinal (GI) bleeding is a common medical emergency and an important cause of morbidity and mortality in high, middle and low income countries. Despite advances in resuscitative, pharmacological and endoscopic therapy, re-bleeding occurs in $10 \%$ of patients with non-variceal bleeding and up to $25 \%$ of those with variceal bleeding and is an important predictor of death. Excessive fibrinolysis may play an important role both in the failure to control initial bleeding and in the precipitation of re-bleeding through premature breakdown of blood clots at sites of vascular injury. This raises the possibility that an antifibrinolytic drug administered following GI bleeding could limit severity of bleeding and transfusion requirements.

Methods HALT-IT has been designed as a large, pragmatic randomised controlled trial which aims to quantify the efficacy and safety of tranexamic acid (TXA) in adults with significant acute upper or lower gastrointestinal bleeding. The trial will determine the effect of early administration of TXA on mortality, morbidity, blood transfusion, surgical intervention and health status in patients with GI bleeding. The primary outcome is death in hospital within 28 days of randomisation. Secondary outcomes include re-bleeding, need for surgery or radiological intervention, blood product transfusion and thromboembolic events.

Results UK recruitment began in August 2013. By January 2014, a total of 507 patients were randomised across 26 actively recruiting sites, averaging a recruitment rate of 20 patients per week. Centralised and statistical data monitoring ensures trial participants meet inclusion criteria and allows real time monitoring of event rates for the primary and secondary outcomes. The results will be presented by intention to treat and a pre-specified subgroup analysis will also determine the treatment effect in patients with liver cirrhosis and variceal bleeding.

Conclusion HALT-IT aims to recruit 8000 participants in hospitals worldwide and recruitment is ahead of schedule based upon a strong performance in the UK. The success of the trial to date has been dependent upon multi-disciplinary and societal engagement as well as infrastructural support provided by NIHR research networks. The results will add to our expanding knowledge about the role of tranexamic acid as an agent for patients with significant bleeding. It is anticipated that the full trial results will be available in 2017.

Disclosure of Interest None Declared.

\section{PTU-186 THE "SPEEDBOAT-RS2": A NEW MULTI-MODALITY ENDOSCOPIC DEVICE FOR GASTRIC AND OESOPHAGEAL SUBMUCOSAL DISSECTION AND TUNNELLING}

${ }^{1}$ ZP Tsiamoulos ${ }^{*},{ }^{2} \mathrm{C}$ Hancock, ${ }^{3} \mathrm{PD}$ Sibbons, ${ }^{4} \mathrm{LA}$ Bourikas, ${ }^{1} \mathrm{BP}$ Saunders. ${ }^{1}$ Wolfson Unit for Endoscopy, St Mark's Hospital/Academic Institute, London, UK; ${ }^{2}$ Department of Electronic Engineering, Bangor University, Bangor; ${ }^{3}$ Department of Surgical Sciences, Northwick Park Institute for Medical Research, London, UK; ${ }^{4}$ Department of Gastroenterology, University of Crete, Crete, Greece

\subsection{6/gutjnl-2014-307263.260}

Introduction Gastric and oesophageal mucosal lesions are optimally removed en-bloc for accurate histology and complete resection. We describe, a simple to use, multi-modality endoscopic device (“Speedboat-RS2") for en-bloc gastric/oesophageal mucosal resection and for oesophageal submucosal tunnelling. Methods The 'Speedboat-RS2' cuts in forward, lateral and oblique planes using bipolar radio frequency (RF) cutting, provides haemostasis with microwave coagulation and incorporates a retractable needle for submucosal injection and tissue irrigation. The instrument blade has an insulated 'hull' to prevent thermal injury to the muscularis propria and the device catheter is partially torque stable allowing rotation and orientation of the hull 
parallel to the muscle layer. Gastric submucosal dissection and oesophageal submucosal resections/tunnelling procedures were performed on 5 consecutive $60 \mathrm{~kg}$ pigs. All cases were video recorded. The time taken to complete resection/tunnelling, complications encountered and power settings used were recorded. Two animals were euthanized immediately (termination study TS) and three animals were recovered for 3 days (survival study $=\mathrm{SS}$ ). Submucosal defects and excised flaps were measured and assessed histologically.

Results Five (3TS, 2SS) consecutive gastric submucosal dissections, 5 oesophageal resections $\{4$ (3TS, 1SS) semi and 1 (SS) full circumferential oesophageal mucosal resections $\}$ and 2 submucosal tunnelling procedures $\{1$ (SS) with partial myotomy and 1 (TS) with no myotomy $\}$ were performed. The median time to complete a gastric resection was 46 min range (21-83min) using RF cutting $35 \mathrm{~W}$ and 41 min range (12-50 min) using RF cutting $25 \mathrm{~W}$ for the oesophageal excision/tunnelling procedure. Median gastric defect size was $55 \mathrm{~mm}$, range $35-70 \mathrm{~mm}$ and median oesophageal defect size was $47 \mathrm{~mm}$, range $35-70 \mathrm{~mm}$. Microwave coagulation was applied for either minor bleeding or visible vessels on 57 occasions (mean energy 7.5 W). No endoscopic or histologic perforations were noted. All excised flaps were appropriate for histological assessment apart from one oesophageal flap that was mildly heat damaged. Gastric and oesophageal muscle layers/serosa were intact and viable. In three oesophageal cases, there was a mild muscle cell alteration but contiguity was retained. In one gastric resection, another dissection knife assisted the last ribbon cut.

Conclusion This initial evaluation of "Speedboat-RS2" in the upper GI tract suggests that it facilitates rapid and safe en-bloc mucosal resection in the oesophagus and stomach. It also appears promising for safe and rapid submucosal tunnelling in the oesophagus and has potential to be utilised for POEM.

Disclosure of Interest Z. Tsiamoulos Consultant for: Creo Medical Ltd, C. Hancock Shareholder of: Creo Medical Ltd, P. Sibbons Paid instructor for: Creo Medical Ltd, L. Bourikas: None Declared, B. Saunders: None Declared.

\section{Colon and anorectum}

\section{PWE-001 FIELD CANCERISATION THEORY IN COLORECTAL CANCER (CRC): WHAT ROLE DO FIBROBLAST GROWTH FACTORS HAVE?}

${ }^{1} \mathrm{~A}$ Patel ${ }^{*},{ }^{2} \mathrm{~N}$ Williams, ${ }^{3} \mathrm{C}$ Nwokolo, ${ }^{1} \mathrm{G}$ Tripathi, ${ }^{1,3} \mathrm{R}$ Arasaradnam. ${ }^{1} \mathrm{CSR}$, University of Warwick, UK; ${ }^{2}$ Colorectal Surgery, University Hospital Coventry and Warwickshire, Coventry, UK; ${ }^{3}$ Gastroenterology, University Hospital Coventry and Warwickshire, Coventry, UK

\subsection{6/gutinl-2014-307263.261}

Introduction Characterisation of the molecular field defect around colorectal cancer (CRC) could enable identification of novel biomarkers that could be used for early detection of CRC. Previous studies have suggested fibroblast growth factor 19 (FGF19) may play a role in CRC formation through interaction with the B-catenin/wnt signalling cascade. The role of fibroblast growth factor 7 (FGF7) however remains controversial. The aim of this study was to determine if there are differences in FGF19 and FGF7 gene expression in cancer tissue and the adjacent "normal tissue' compared with normal colonic tissue.

Methods Mucosal pinch biopsies were taken from the rectum and caecum at time of colonoscopy for healthy controls. For CRC patients, tissue samples were taken from the tumour, adjacent to the tumour and at the resection margin of the colectomy specimen. Healthy controls were age and sex matched to CRC patients. Quantitative real time PCR was used to determine gene expression of FGF19, its receptor FGFR4, FGF7 and its receptor, FGFR2. Results were further validated using immunohistochemistry. Serum levels of FGF19 were measured using the Quantikine ELISA kit (RandD systems, UK).

Results 49 patients were recruited (28 M: $21 \mathrm{~F}$, median age 71 years (range 48-86 years)); 18 patients with CRC and 32 healthy controls. There was no overall difference in gene expression of FGF19/FGFR4 or FGF7/FGFR2 between cancer patients and healthy controls. There was upregulation of FGFR4 in mucosa adjacent to the tumour (mean fold change 1.23 vs. 0.93 , $p=0.38$ ) and the tumour itself (mean fold change 1.49 vs. $1.04, \mathrm{p}=0.700$ ) in patients whose tumour expressed FGF19 compared to those that did not. Patients with upregulation of FGF19/FGFR4 had a significantly lower fasting serum FGF19 level $(119 \mathrm{pg} / \mathrm{ml}$ versus $208 \mathrm{pg} / \mathrm{ml}, \mathrm{p}=0.05)$.

FGF7 was upregulated in 6/19 cancers; this was associated with a significant upregulation in FGF7 in adjacent mucosa compared with cancers where FGF7 was downregulated (mean fold change 3.62 vs. $0.95, p=0.018$ ). There was a non-significant trend towards upregulation of the receptor (FGFR2) in mucosa adjacent to the cancer and the tumour tissue itself.

Conclusion Upregulation of FGFR4 in patients whose tumours expressed FGF19 corresponded inversely with serum FGF19 suggesting its potential as a putative biomarker. Significant upregulation of FGF7 in 'normal' mucosa adjacent to only tumours that express FGF7 lends support to the field theory of colorectal carcinogenesis.

Disclosure of Interest None Declared.

\section{PWE-002 THE POSITIVE PREDICTIVE VALUE OF A COLONOSCOPIST LABELLING A LESION AS CANCER - WHAT SHOULD WE TELL THE PATIENT?}

AM Verma*, RE Smith, A Dixon, AP Chilton. Gastroenterology, Kettering General Hospital NHS Foundation Trust, Kettering, UK

\subsection{6/gutjnl-2014-307263.262}

Introduction Colonoscopy is the modality of choice for bowel cancer screening and investigation of iron deficiency anaemia. Hence colonoscopists are most likely to diagnose colorectal cancer (CRC). It is desirable to inform a patient post endoscopy they have CRC but a colonoscopist may fear giving an incorrect diagnosis.

All patients with CRC are discussed at the weekly multi-disciplinary team meeting. A delay in treatment is often caused by the patient not being aware of a CRC diagnosis. This may require an additional appointment to inform the patient before the appointment with a Surgeon or Oncologist to discuss treatment.

This delay can increase the risk of progression of CRC and reduces the time the patient and family have to adjust to a CRC diagnosis and its consequences. We aim to test the positive predictive value (PPV) of colonoscopists diagnosing CRC and audit if patients were informed.

Methods 8561 colonoscopies undertaken at Kettering General Hospital (KGH), if "tumour/cancer" was recorded this was correlated to outcome. The reporting software gives an option to record if patient "informed of cancer" or "informed of lesion". Results "Tumour/cancer" recorded 350 times $(4.09 \%$ of colonoscopies) 